\title{
Applying negative pressure wound therapy in associating liver partition and portal vein ligation for staged hepatectomy: A case report
}

\author{
BO ZHAI ${ }^{1}$, XIN JIN $^{1}$, RUI WANG $^{2}$, TAISHI FANG ${ }^{1}$, JING QU $^{3}$, YING WANG $^{4}$, MING LIU $^{1}$ and LISHAN XU $^{1}$ \\ ${ }^{1}$ Department of General Surgery, The Fourth Affiliated Hospital of Harbin Medical University, \\ Harbin, Heilongjiang 150001; ${ }^{2}$ Department of Gastroenterology, Heilongjiang Provincial Hospital, \\ Harbin, Heilongjiang 150036; Departments of ${ }^{3}$ Orthopedics and ${ }^{4}$ Anesthesiology, The Fourth \\ Affiliated Hospital of Harbin Medical University, Harbin, Heilongjiang 150001, P.R. China
}

Received March 10, 2016; Accepted March 3, 2017

DOI: $10.3892 / e t m .2017 .4566$

\begin{abstract}
Staged hepatectomy, particularly associating liver partition and portal vein ligation for staged hepatectomy (ALPPS) is a recently developed advanced surgery for resecting liver tumors. However, large wound surfaces, incomplete drainage and high rates of mortality are vital limitations of ALPPS. The present case study describes a patient with liver cancer who underwent ALPPS surgery combined with negative pressure wound therapy. A 46-year-old male patient was hospitalized due to right upper abdominal pain. Computed tomography and magnetic resonance cholangiopancreatography scan results indicated gallbladder cancer, accompanied by multiple liver metastases. Resection of the right trisegment and partial resection of metastatic nodules in the left outer lobe were performed. Vacuum sealing drainage was pre-implanted at liver cross-sections with drainage tubes to drain the bile. The patient exhibited improved wound healing compared with conventional ALPPS.
\end{abstract}

\section{Introduction}

Liver cancer is the sixth most common cancer worldwide with varying geographic distribution. High incidence rates have been reported in developing countries, particularly in China ( 26 per 100,000 people) when compared with developed countries ( 10 per 100,000 people) (1). Early diagnosis of liver cancer remains a major obstacle and typically results in poor

Correspondence to: $\mathrm{Dr}$ Lishan $\mathrm{Xu}$, Department of General Surgery, The Fourth Affiliated Hospital of Harbin Medical University, 37 Yiyuan Street, Harbin, Heilongjiang 150001, P.R. China

E-mail:1sxu6688@sina.com

Key words: liver cancer, staged hepatectomy, associating liver partition and portal vein ligation for staged hepatectomy, negative pressure wound therapy, vacuum sealing drainage outcomes after clinical intervention (2). Resection of tumors is a common intervention to increase the survival of patients (3). However, large tumors within the liver and multiple metastases require complete resection in order to improve the long-term survival of patients, and numerous approaches have been attempted to achieve complete resection (3). Staged hepatectomy and approaches to induce hypertrophy, including percutaneous portal vein embolization and intraoperative portal vein ligation, are recent advances in liver surgery (4). Schnitzbauer et al (5) indicated that a two-staged hepatectomy was performed for the first time in a patient with hilar cholangiocarcinoma in 2007 and that associating liver partition and portal vein ligation for staged hepatectomy (ALPPS), a surgical approach for liver cancer with excessive tissue resection, was first described in 2012. However, ALPPS surgery has several limitations, including a large wound surface, incomplete drainage and high mortality rates $(5,6)$. Acute liver and renal failure and infectious complications have been identified as predominant causes for mortality (6). Numerous modifications to the originally described ALPPS approach have been reported (7). Previous studies have demonstrated that careful selection of specific patients for ALPPS resulted in reduced or no associated mortality $(5,6)$. In February 2015, the first international consensus meeting concerning ALPPS was conducted in Germany, and the following recommendations were made via patient selection and inclusion criteria: ALPPS would only be considered for patients requiring right trisectionectomy, patients with hepatocellular carcinoma or cholangiocarcinoma and patients with extensive bilobar colorectal liver metastases requiring an extended hepatectomy (7). Furthermore, in cases where portal vein embolization or portal vein ligation failed, ALPPS may be an alternative surgery. Hence, ALPPS is a novel surgical technique that has been indicated for use in specific patients with liver cancer.

Negative pressure wound therapy (NPWT; also known as vacuum-assisted closure) is a therapeutic approach that utilizes a vacuum dressing to promote the healing of large wounds and vacuum sealing drainage (VSD; Wuhan VSD Medical Science $\&$ Technology Corp., Wuhan, China) is one of the novel NPWT techniques (8). In VSD, the commonly used polyurethane 
material is replaced by polyvinyl alcohol (PVA) (9). PVA has higher tissue compatibility and tensile strength that does not result in debris shedding or stick to surrounding tissues (9). Considering the aforementioned characteristics of PVA, the VSD technique may be used to improve wound healing in ALPPS surgery. Hence, the present case report describes a patient with liver cancer who underwent ALPPS surgery and co-application of NPWT to heal the large wound and improve the outcome of therapeutic intervention.

\section{Case report}

Ethical statement. The present case study was approved by the Ethics Committee of The Fourth Affiliated Hospital of Harbin Medical University (Harbin, China). Written informed consent was obtained from the patient.

Presenting concerns. In November 2014, a 46-year-old male patient was hospitalized in the Department of General Surgery at The Fourth Affiliated Hospital of Harbin Medical University (Harbin, China) due to right upper abdominal pain with no symptoms of jaundice. The patient had no disease history or treatment history related to the liver prior to this hospitalization.

Diagnostic focus and assessment. Routine blood test results revealed the following: White blood cell count, $6.1 \times 10^{9} / 1$ (reference range: $3.5-9.5 \times 10^{9} / 1$ ); neutrophils, $69.9 \%$ (reference range: $40-75 \%)$; red blood cell count, $5.02 \times 10^{12} / 1\left(3.8-5.1 \times 10^{12} / 1\right)$; hemoglobin, $155 \mathrm{~g} / 1$ (reference range: $115-150 \mathrm{~g} / \mathrm{l}$ ); and hematocrit, $46.3 \%$ (reference range: $35-50 \%$ ). Liver function test results revealed the following: Total bilirubin, $264.6 \mu \mathrm{mol} / 1$ (reference range: 3.1-22.5 $\mu \mathrm{mol} / 1$ ); aspartate aminotransferase, $48 \mathrm{U} / 1$ (reference range: 13-35 U/1); alanine aminotransferase, $120 \mathrm{U} / 1$ (reference range: 7-40 U/1); and albumin, $38.6 \mathrm{~g} / \mathrm{l}$ (reference range: $40-55 \mathrm{~g} / \mathrm{l}$ ). The patient's carbohydrate antigen-199 level was 1,000 U/ml (reference range: 0-27 U/ml) and the $\alpha$-fetoprotein level was $2,048 \mathrm{ng} / \mathrm{ml}$ (reference range: 13-150 ng/ml). Computed tomography scan and magnetic resonance cholangiopancreatography scan results indicated gallbladder cancer accompanied by multiple liver metastases including segments V, VIII and IV of the liver. Right posterior lobe metastases were observed in the vicinity of the portal and hepatic veins (Fig. 1A-D).

Therapeutic focus and assessment. Liver volume was calculated using liver resection simulation in the 3D+Medical Imaging Visualization system (Yorktal Digital Medical Imaging Technology, Co., Ltd., Shenzhen, China). The total liver volume, residual liver volume (RLV), and tumor volume were calculated. The RLV was calculated as the total liver volume minus the tumor volume. The RLV was $460.95 \mathrm{ml}$ (18.1\% of the total liver volume; Fig. 1E). Subsequently, resection of the right trisegment was performed. Prior to surgery, intense glucose therapy (50\% glucose solution containing $100 \mathrm{~g}$ of glucose daily for 2 days; CR Double-Crane Pharmaceuticals Co., Ltd., Beijing, China) was administered to the patient to increase the liver glycogen stores and vitamin $\mathrm{K}$ (vitamin $\mathrm{K} 1$, $10 \mathrm{mg}$, and vitamin $\mathrm{K} 3,8 \mathrm{mg}$ daily via intramuscular injection for 3 days; Cisen Pharmaceutical Co., Ltd., Jining, China) was administered to improve blood coagulation. Furthermore, reduced glutathione $(1,800 \mathrm{mg} /$ day intravenously for 5 days; YaoPharma Co., Ltd., Chongqing, China) and magnesium isoglycyrrhizinate (200 mg intravenous daily for 5 days; Chia Tai Tianqing Pharmaceutical Group Co., Ltd., Lianyungang, China) were also administered to protect liver function.

The first surgery was performed in November 2014. The common bile duct along the lateral part of duodenum was disarticulated and the hepatoduodenal ligament was skeletonized. Three metastatic nodules in segments II and III of the left outer lobe of the liver were partially resected. A liver portion between segment IV and segments II-III was disarticulated. Following this, the portal vein and hepatic artery branches connected to segment IV were ligated, the right portal vein was ligated, and the bile duct at left hepatic duct confluence was disarticulated. Subsequently, a Y-tube was inserted to drain the bile out of the body. Developer agent (fat emulsions; B. Braun Melsungen AG, Melsungen, Germany) was injected into the Y-tube to examine the bile leakage at the liver cross-section (Fig. 1F-J). A VSD dressing was placed on the resected surface of the liver, with inferior and anterior margins $1 \mathrm{~cm}$ beyond the resection surface. It had two drainage tubes $3 \mathrm{~cm}$ apart. When a secondary surgery was not feasible, the VSD dressing could be removed through the $3-\mathrm{cm}$ incision between the two tubes (Fig. 2). Moreover, two normal drainage tubes were also placed at the liver cross-section. One tube was placed at the hepatorenal recess (the lowest point of the supine position) and the other was placed behind the liver duodenum ligament to drain the residual effusion at the cross section and small omentum, respectively. Post-surgery, continuous negative pressure suction was applied via VSD at $50 \mathrm{kPa}$ and an intermittent saline flush (50 $\mathrm{ml}$ every hour) was performed through a flush pipe. No negative pressure suction was performed for the normal drainage tubes. The patient received prophylactic antibiotic therapy in the form of Ulinastatin (200,000 U/day intravenously for 5 days; Techpool Bio-Pharma Co., Ltd., Guangzhou, China) and Omegaven (100 ml/day intravenously for 5 days postoperative; Fresenius Kabi Asia-Pacific, Ltd., Wanchai, Hong Kong) as anti-inflammatory medications, and proteins (albumin, $20 \mathrm{~g}$ /day intravenously for 3 days; Shanghai RAAS Blood Products Co., Ltd., Shanghai, China) as treatment to protect liver function. A total of 7 days after the first surgery, remnant liver volume was increased to $117 \%$ of the original volume, accounting for 39.3\% (Fig. 3A and B) of the standard liver volume.

The second surgery was performed 8 days after the first surgery and the abdomen was accessed along the original incision. On investigation, the volume of the left outer lobe was notably increased and the VSD duct was appropriately fixed on the liver cross section (adhered to both sides of the liver cross section). The adhesion was slight and easy to separate. No effusion was observed surrounding the VSD duct and no bleeding occurred at the liver cross section when VSD was removed. Furthermore, the perihepatic ligament was liberated and the right hepatic artery and right hepatic vein were ligated and excised. The hepatic vein was disarticulated below the confluence point of the middle hepatic and left hepatic veins and the broken ends were sutured. The left outer lobe bile duct was disarticulated, the right liver trisegment was resected and Roux-Y anastomosis was achieved on the left outer lobe bile 

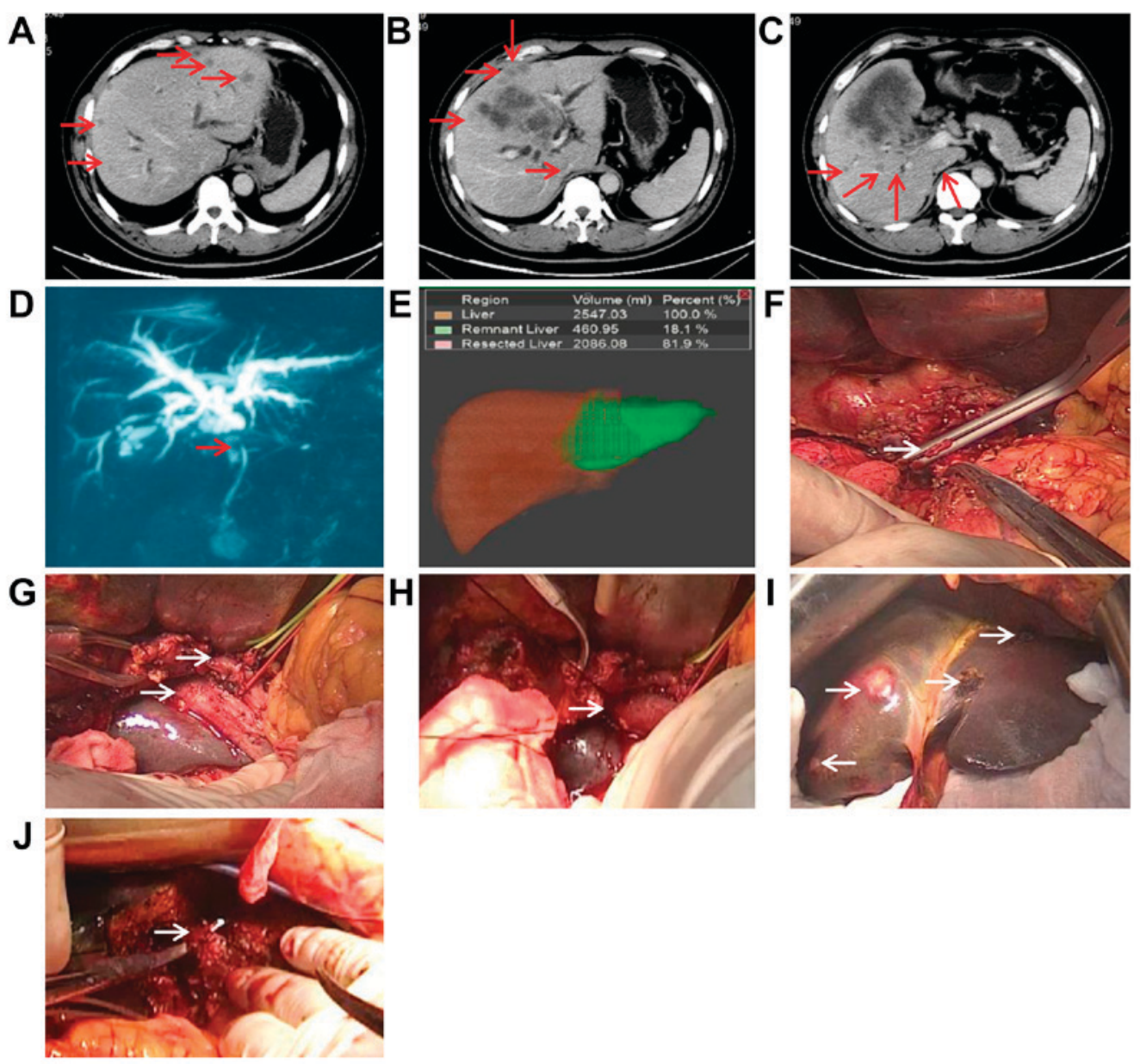

Figure 1. Preoperative CT (and future liver remnant=18.1\%) and intraoperative findings before VSD implanting. (A-C) Preoperative CT with metastasis sites indicated (arrows). (D) Magnetic resonance cholangiopancreatograpy with the involved hepatic duct and dilated intrahepatic biliary duct indicated (arrow). (E) Calculation of the residual liver volume. (F) Resection of the common bile duct (arrow). (G) Isolation of the hepatoduodenal ligament. Red arrow, right hepatic artery; white arrow, right branch of portal vein. (H) Transection of the right branch of portal vein (arrow). (I) Resection of the left lateral lobe metastasis (arrows). (J) Transection of the left medial branch of portal vein (arrow). CT, computed tomography.

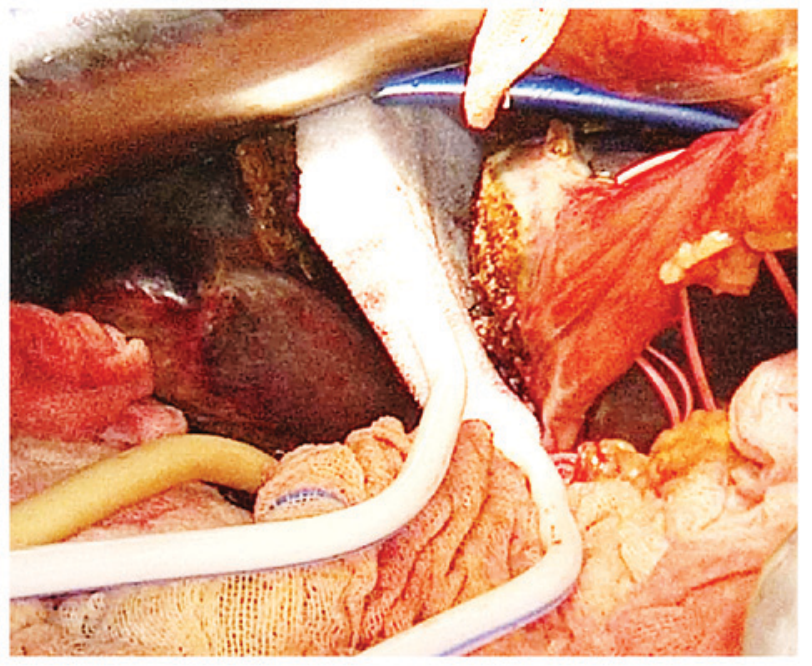

Figure 2. Vacuum sealing drainage implanting and distance between two drainage tubes.

duct and jejunum. One drainage tube was retained at the lower cross section (Fig. 3C-H).
Outcome. The patient exhibited improved wound healing after the application of VSD compared with conventional ALPPS surgery. There was positive improvement in wound adhesion and effusion drainage. The VSD dressing reduced intraoperative abdominal adhesion and effusion and protected the residual liver from tumor invasion of the diseased liver. Previous studies support that the conventional ALPSS method is disadvantageous when used with or without a plastic bag $(6,7)$. Indeed, it is difficult to completely drain the fluid from the plastic bag. Both non-treatment and fibrin glue application present the problem of incomplete drainage and the tumors proximal to the resection surface may invade the residual liver.

This patient was followed up until December 2016, and no evidence of recurrence or metastasis was observed.

\section{Discussion}

With the improvements in technology and surgical techniques, major hepatectomies may be performed with low morbidity and mortality. In the present case report, a two-staged hepatectomy (ALPPS) was performed in a patient with liver cancer and VSD was implanted to improve surgical wound 
A

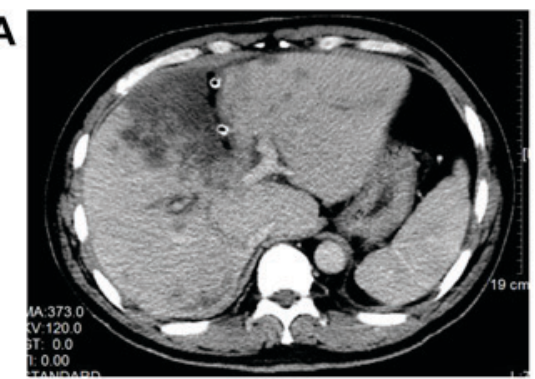

D

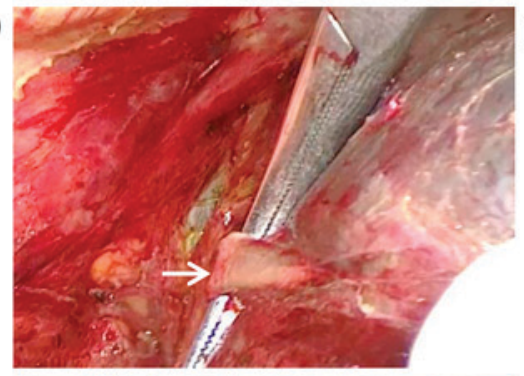

G

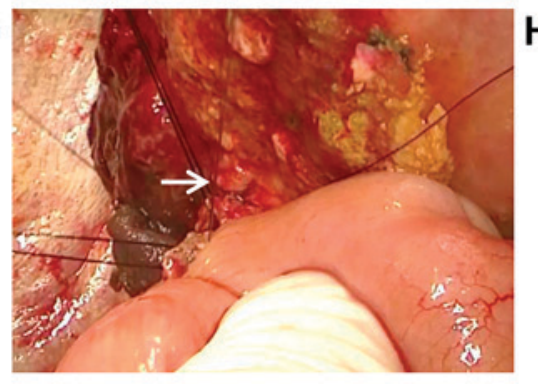

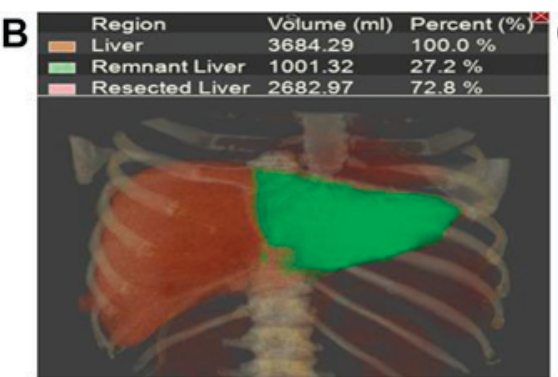

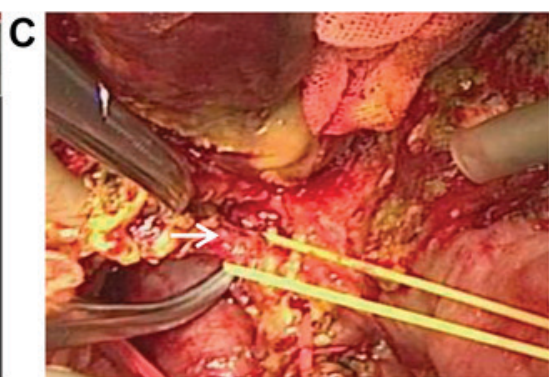

E

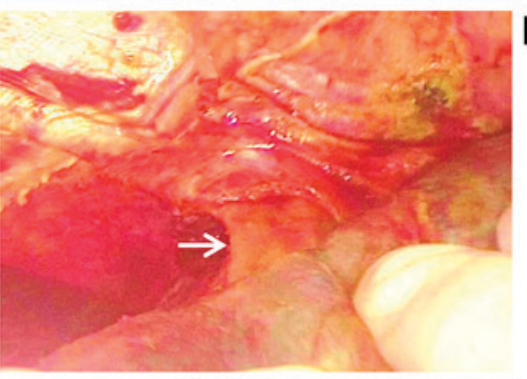

$\mathbf{F}$

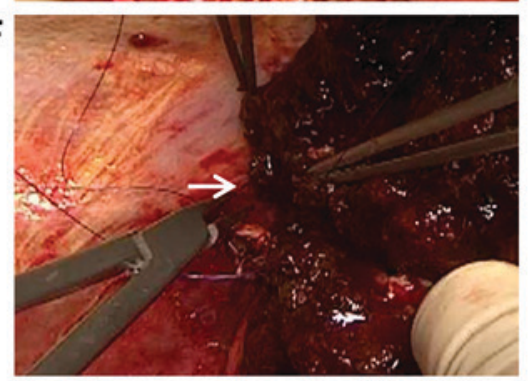

$\mathrm{H}$

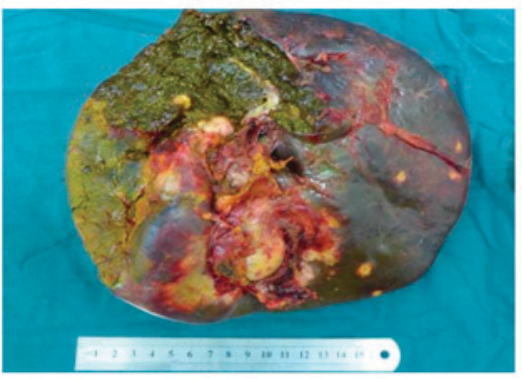

Figure 3. Preoperative computed tomography (remnant liver volume, 39.3\%) and intraoperative findings of the second operation. (A) Left lateral lobe volume of the first liver segment was markedly increased at postoperative day 6. (B) Calculation of the residual liver volume. (C) Transection of the right hepatic artery (arrow). (D) Transection of the perihepatic ligament and hepatic segment vein (arrow). (E) Transection of the right hepatic vein (arrow). (F) Transection of the middle hepatic vein (arrow). (G) Choledochojejunostomy. Arrow, bilioenteric anastomosis. (H) Resection of the specimens.

healing. Results indicated that wound healing in the patient was improved after the application of VSD compared with conventional ALPPS surgery.

Application of VSD in ALPPS surgeries. To the best of our knowledge, the application of VSD to treat abdominal surgical wounds has not yet been reported. VSD dressing has been rarely used for abdominal surgeries because it is non-absorbable. Since it is also large and not smooth, it cannot be pulled out as with a drainage tube; a secondary surgery is required to remove a VSD dressing. In ALPPS surgery, the dressing may be removed during the second surgery. Furthermore, the application of a VSD dressing in this surgery alleviates abdominal inflammation, reduces tissue adhesion and improves the difficulty of secondary surgery. Notably, the placement of a VSD dressing between the diseased and residual parts of the liver prevents tumor invasion. The authors of the present study suggest that the two drainage tubes of the VSD dressing are placed beneath the skin, $3 \mathrm{~cm}$ apart. Therefore, when a secondary surgery is not feasible, it is possible to incise the skin and remove the VSD dressing under local anesthesia. It overcomes the disadvantage of the plastic bag, which is difficult to remove. In the present case report these challenges were overcome effectively. First, VSD was removed without difficulty as the second surgery was performed $\sim 1$ week after ALPPS. Therefore, as the interval between the two surgeries was short, the obtained adhesion was relatively limited and VSD limited the extent of the adhesions. In conclusion, the present case study indicated that the use VSD combined with ALPPS surgery for a patient with liver cancer resulted in improved recovery and wound healing.

Use of VSD in ALPPS surgery. In the present study, VSD was pre-implanted appropriately and fixed on both sides of the liver cross section. To enable complete suction, the front of the upper bound of VSD was outplaced $1 \mathrm{~cm}$ above the liver cross section. Two VSD drainage tubes were placed $3 \mathrm{~cm}$ apart in order to enable easy accessibility during the second surgery and easy removal, if required.

Negative pressure applied at $50 \mathrm{kPa}$. Excessive negative pressure may increase the bleeding risk and may result in the attraction of intestine and omentum tissues, leading to wrapping and adhesions (10). Thus, a low negative pressure of 50 $\mathrm{kPa}$ was chosen in the present case. However, specific factors should be considered when choosing an appropriate negative pressure. Firstly, pressure should be high enough to resist the resistance of VSD sponge material to ensure that the negative pressure may suck the liquid surrounding VSD; continuous negative pressure suction and syphonage effect of sponges allow for more complete drainage (8-10). Secondly, if the pressure is too low, the sponge becomes too wet, which affects the 
adhesion properties of the sponge. Hence, choosing an optimal negative pressure is important to improve the functionality of VSD.

Surgeons performed ALPPS along with VSD on another patient who was diagnosed with Bismush type IV hilar cholangiocarcinoma. In September 2015, a 59-year-old male patient was hospitalized at the Department of General Surgery at The Fourth Affiliated Hospital of Harbin Medical University (Harbin, China) due to symptoms of jaundice. White blood cell count was $3.7 \times 10^{9} / 1$; neutrophils, $63.1 \%$; red blood cell count, $4.5 \times 10^{12} / 1\left(3.8-5.1 \times 10^{12} / 1\right)$; hemoglobin, $120 \mathrm{~g} / 1$; and hematocrit, $41.1 \%$. Liver function test results revealed the following: Total bilirubin, $383.6 \mu \mathrm{mol} / 1$; aspartate aminotransferase, $72 \mathrm{U} / 1$; alanine aminotransferase, $100 \mathrm{U} / 1$; and albumin, $41.8 \mathrm{~g} / 1$ The patient's carbohydrate antigen-199 level was $1,000 \mathrm{U} / \mathrm{ml}$. A percutaneous transhepatic cholangial drainage tube was placed preoperatively at the right hepatic lobe to drain the bile. It was found that tumors had invaded the right hepatic duct and adjacent tissues, which indicated the necessity of right hemi-liver resection. However, as treatment for jaundice had not been performed on the left hemi-liver, the risk of postoperative liver function failure was relatively high. Thus, ALPPS was performed on the right hemi-liver. As biliary-enteric anastomosis was performed at the same time, conventional plastic bags, VSD joint wound protection and suction approaches were adopted to prevent bile leakage and adhesion.

The present case report has certain limitations. VSD material must be improved in order to obtain satisfactory adhesion to tissues and improve the suction of bile wastes. Certain factors, including the selection of optimal negative pressure, VSD material and interval time between staged hepatectomies require further investigation. Moreover, large-scale randomized trials are required to demonstrate the safety, efficacy and long-term outcomes of this approach.

In conclusion, in cases where patients that require conventional two-staged hepatectomies do not fulfill the criteria to undergo resection due to insufficient hypertrophy or tumor progression, ALPPS may be used as a salvage procedure, despite the risk of increased morbidity and possible mortality. As a wound protection and drainage technique, VSD may be employed in ALPPS surgeries in order to decrease effusion and adhesion complications. In the meantime, its application in alternative abdominal surgeries remains to be established.

\section{Acknowledgements}

The present study was supported in part by the National Natural Scientific Foundation of China (grant 81401975), Natural Science Foundation of Heilongjiang Province (grant no. D201250), China Postdoctoral Science Foundation (grant no. 2016M591564), Heilongjiang Postdoctoral Fund (grant no. LBH-Z14219 and LBH-Z16191), Scientific Research Project of Heilongjiang Medical Science Institute (grant no. 201502 and 201710), and Heilongjiang Province Health Bureau (grant no. 2014-399).

\section{References}

1. Ananthakrishnan A, Gogineni V and Saeian K: Epidemiology of primary and secondary liver cancers. Semin Intervent Radiol 23: 47-63, 2006.

2. Yuen MF, Cheng CC, Lauder IJ, Lam SK, Ooi CG and Lai CL: Early detection of hepatocellular carcinoma increases the chance of treatment: Hong Kong experience. Hepatology 31: 330-335, 2000.

3. Bruix J and Sherman M; American Association for the Study of Liver Diseases: Management of hepatocellular carcinoma: An update. Hepatology 53: 1020-1022, 2011.

4. Della Sala S: A daguerreotype of Phineas Gage? Cortex 47: 415, 2011.

5. Schnitzbauer AA, Lang SA, Goessmann H, Nadalin S, Baumgart J, Farkas SA, Fichtner-Feigl S, Lorf T, Goralcyk A, Hörbelt R, et al: Right portal vein ligation combined with in situ splitting induces rapid left lateral liver lobe hypertrophy enabling 2-staged extended right hepatic resection in small-for-size settings. Ann Surg 255: 405-414, 2012.

6. Torres OJ, Fernandes Ede S, Oliveira CV, Lima CX, Waechter FL, Moraes-Junior JM, Linhares MM, Pinto RD, Herman P and Machado MA: Associating liver partition and portal vein ligation for staged hepatectomy (ALPPS): The Brazilian experience. Arq Bras Cir Dig 26: 40-43, 2013 (In English, Portuguese).

7. Zhang GQ, Zhang ZW, Lau WY and Chen XP: Associating liver partition and portal vein ligation for staged hepatectomy (ALPPS): A new strategy to increase resectability in liver surgery. Int J Surg 12: 437-441, 2014.

8. Banasiewicz T, Borejsza-Wysocki M, Meissner W, Malinger S, Szmeja J,Kościński T, Ratajczak A and Drews M: Vacuum-assisted closure therapy in patients with large postoperative wounds complicated by multiple fistulas. Wideochir Inne Tech Maloinwazyjne 6: 155-163, 2011.

9. Hu N, Wu XH, Liu R, Yang SH, Huang W, Jiang DM, Wu Q, Xia T, Shao ZW and Ye ZW: Novel application of vacuum sealing drainage with continuous irrigation of potassium permanganate for managing infective wounds of gas gangrene. J Huazhong Univ Sci Technolog Med Sci 35: 563-568, 2015.

10. Ren H and Li Y: Severe complications after negative pressure wound therapy in burned wounds: Two case reports. Ther Clin Risk Manag 10: 513-516, 2014. 